This item was submitted to Loughborough's Research Repository by the author.

Items in Figshare are protected by copyright, with all rights reserved, unless otherwise indicated.

\title{
MVMO-based approach for optimal placement and tuning of supplementary damping controller
}

\section{PLEASE CITE THE PUBLISHED VERSION}

http://dx.doi.org/10.4314/ijest.v7i3.12S

\section{PUBLISHER}

(C) MultiCraft

\section{VERSION}

VoR (Version of Record)

\section{PUBLISHER STATEMENT}

This work is made available according to the conditions of the Creative Commons Attribution-NonCommercialNoDerivatives 4.0 International (CC BY-NC-ND 4.0) licence. Full details of this licence are available at: https://creativecommons.org/licenses/by-nc-nd/4.0/

\section{LICENCE}

CC BY-NC-ND 4.0

\section{REPOSITORY RECORD}

Torres, J.L. Rueda, and Francisco M. Gonzalez-Longatt. 2019. "Mvmo-based Approach for Optimal Placement and Tuning of Supplementary Damping Controller". figshare. https://hdl.handle.net/2134/19795. 


\section{INTERNATIONAL \\ JOURNAL OF \\ ENGINEERING, SCIENCE \\ AND TECHNOLOGY}

www.ijest-ng.com

www.ajol.info/index.php/ijest

(C) 2015 MultiCraft Limited. All rights reserved

\title{
MVMO-based approach for optimal placement and tuning of supplementary damping controller
}

\author{
J. L. Rueda Torres ${ }^{1 *}$, F. Gonzalez-Longatt ${ }^{2}$ \\ ${ }^{1 *}$ Department of Electrical Sustainable Energy, Delft University of Technology, THE NETHERLANDS \\ ${ }^{2}$ Centre for Renewable Energy Systems Technology (CREST), Loughborough University, UNITED KINGDOM \\ "Corresponding Author: e-mail: j.l.ruedatorres@tudelft.nl, Tel +31-1527-86239, Fax.+31-1527-2590063
}

\begin{abstract}
This paper introduces an approach based on the Swarm Variant of the Mean-Variance Mapping Optimization (MVMO-S) to solve the multi-scenario formulation of the optimal placement and coordinated tuning of power system supplementary damping controllers (POCDCs). The effectiveness of the approach is evaluated based on the classical IEEE 39-bus (New England) test system. Numerical results include performance comparisons with other metaheuristic optimization techniques, namely, comprehensive learning particle swarm optimization (CLPSO), genetic algorithm with multi-parent crossover (GA-MPC), differential evolution DE algorithm with adaptive crossover operator, linearized biogeography-based optimization with reinitialization (LBBO), and covariance matrix adaptation evolution strategy (CMA-ES).
\end{abstract}

Keywords: Coordinated control, damping controllers, metaheuristics, small-signal stability.

DOI: http://dx.doi.org/10.4314/ijest.v7i3.12S

\section{Introduction}

Traditionally, the placement and tuning of power system damping controllers have been tackled as individual problems based on participation factors, residues, damping torque, sensitivity coefficients and singular value decomposition (Karimpour et al, 2005; Pagola et al, 1989; Wang et al, 1997). Alternatively, the simultaneous solution for both tasks has also been investigated from optimization problem point of view. Particularly, the joint determination of optimal placement and coordinated tuning of power system damping controllers (OPCDC) constitutes a challenging optimization problem due to the mix-integer combinatorial nature as well as to the nonlinearity, multimodality, and no convexity of the search space (Sebaa et al, 2010).

Previously reported approaches based on modified versions of genetic algorithm (Sebaa et al, 2009), particle swarm optimization (Eslami et al, 2009), and differential evolution (Wang et al, 2009) highlight the potential of metaheuristic optimization algorithms for solving the OPCDC. Due to the stochastic nature of the underlying evolutionary mechanism, further research is needed to ascertain the robustness of these algorithms, which also motivates the application and extension of emerging metaheuristic optimization algorithms. This paper presents an approach based on the Swarm Mean-Variance Mapping optimization (MVMO-S), which extends the single-solution variant of MVMO to a population based strategy. To achieve efficient and fast search capability, MVMO-S, utilizes swarm intelligence precepts and a multi-parent crossover criterion. The rest of the paper is organized as follows: Section II presents the formulation of the OPCDC and overviews the main features of the MVMO-S algorithm. Section II provides a case study on the IEEE New England 39 bus test system. Finally, conclusions and outlook for future research are summarized in Section IV

\section{Theoretical Background}

2.1. Optimization problem statement

Mathematically, the OPCDC problem has the following format (Rueda et al, 2014): 
Minimize

$$
\mathrm{OF}=\left(\zeta_{\mathrm{sys}}^{*}-\zeta_{\mathrm{th}}\right)^{2}+\mathrm{w}_{1}\left(\alpha_{\mathrm{sys}}^{*}-\alpha_{\mathrm{th}}\right)^{2}
$$

subject to

$$
\mathbf{x}_{\min } \leq \mathbf{x} \leq \mathbf{x}_{\max }
$$

where $\alpha_{\mathrm{th}}$ is a predefined threshold for minimum acceptable modes' real part (i.e. damping factor). $\zeta_{\mathrm{sys}}^{*}$ and $\alpha_{\mathrm{sys}}^{*}$ correspond to the global damping ratio and damping factor of the system (among the nm critical OMs) throughout nsc representative scenarios. The vector $\mathbf{x}$ constitutes the solution of the problem, so it contains the damping controller's tuning parameters (gains $K_{S D C}$, and time constants $T_{1}$ to $T_{4}$ ), which are continuous variables, and their locations, which are coded by using logical variables. The weighting factor $w_{1}$ is a positive number that is used for combining the squared difference between $\alpha_{\text {sys }}^{*}$ and $\alpha_{\text {th }}$ with the squared difference between $\zeta_{\mathrm{sys}}^{*}$ and $\zeta_{\mathrm{th}} \cdot \zeta_{\mathrm{sys}}^{*}$ and $\alpha_{\mathrm{sys}}^{*}$ are determined as follows:

$$
\begin{aligned}
\zeta_{\mathrm{sys}}^{*} & =\min _{\mathrm{j}=1 \mathrm{~K} n \mathrm{sc}}\left[\min _{\mathrm{k}=1 \mathrm{~K} \mathrm{~nm}}\left(\zeta_{\mathrm{k}}\right)\right] \\
\alpha_{\mathrm{sys}}^{*} & =\max _{\mathrm{j}=1 \mathrm{~K} \mathrm{nsc}}\left[\max _{\mathrm{k}=1 \mathrm{~K} \mathrm{~nm}}\left(\alpha_{\mathrm{k}}\right)\right]
\end{aligned}
$$

\subsection{MVMO-S}

Mean-variance mapping optimization (MVMO) is a recently introduced evolutionary algorithm, which has some basic conceptual similarities to other heuristic approaches, but it constitutes a fundamentally new evolutionary mechanism with two salient features. Firstly, MVMO performs by considering normalized range of the search space for all optimization variables within $[0,1]$. This ensures that new values generated for optimization variables in offspring creation stage are always within their bounds. The optimization variables are de-normalized before every fitness evaluation. Secondly, MVMO exploits the statistical attributes of search dynamics by using a special mapping function for mutation operation on the basis of the mean and variance of the n-best solutions attained so far and saved in a continually-updated archive (Rueda et al, 2013).

The original MVMO represents a single particle approach, which has shown a great potential for solving different optimization problems. This paper presents a new variant of MVMO, termed as MVMO-S, which adopts a swarm intelligence scheme and incorporates a multi-parent crossover strategy to increase the search diversity while striving for a balance between exploration and exploitation. The overall procedure is described as follows:

-Step 1: Define $\mathrm{N}_{\mathrm{p}}$, the initial and final values $\left(f_{\mathrm{s} \_ \text {ini }}^{*}\right.$ and $\left.f_{\mathrm{s}_{-} \text {final }}^{*}\right)$ for scaling factor $f_{\mathrm{s}}$, solution archive size, dynamic shape factor $\Delta d$, the initial and final proportion of good particles $\left(g_{\mathrm{p}_{-} \text {ini }}^{*}\right.$ and $\left.g_{\mathrm{p}_{-} \text {final }}^{*}\right)$, and the initial and final number of dimensions $\left(\mathrm{m}_{\text {ini }}^{*}\right.$ and $\left.\mathrm{m}_{\text {final }}^{*}\right)$ to be selected for mutation operation. Next, generate an initial random population of $\mathrm{N}_{\mathrm{p}}$ particles within the search boundaries and normalize the sampled optimization variables by considering the range of search within $[0,1]$.

-Step 2: De-normalize each particle from $[0,1]$ range to their original [min, max] boundaries and evaluate its fitness.

-Step 3: Fill/update the solution archive associated to each particle. The archive stores the n-best child solutions achieved so far in a descending order of fitness. The archive size is fixed for the entire process. For each particle, an update of its archive takes place only if the new solution is better than those in the archive.

Step 4: The first ranked solutions (i.e. local bests) of all solution archives are classified into two groups: A set of GP "good particles", and the set of remaining $\mathrm{N}_{\mathrm{p}}$-GP "bad particles". Local best-based parent assignment is adopted for each particle classified as good, whereas for each bad particle $\mathbf{x}_{\mathrm{p}}$, the parent $\mathbf{x}_{\mathrm{p}}^{\text {parent }}$ is synthesized by using the following multi-parent criteria.

$$
\mathbf{x}_{\mathrm{p}}^{\text {parent }}=\mathbf{x}_{\mathrm{k}}+\beta\left(\mathbf{x}_{\mathrm{i}}-\mathbf{x}_{\mathrm{j}}\right)
$$

where $\mathbf{x}_{\mathbf{i}}, \mathbf{x}_{\mathbf{j}}$, and $\mathbf{x}_{\mathbf{k}}$ represent the first (global best), the last, and a randomly selected intermediate particle in the group of good particles, respectively. The factor $\beta$ is a random number, which is drawn according to

$$
\beta=0.5-0.25 \cdot \alpha, \quad \alpha=i / i_{\max }
$$

where $i$ denotes fitness evaluation number, and $r_{n}$ is a random number with uniform distribution in [0,1].

An element of $\mathbf{x}_{\mathrm{p}}^{\text {parent }}$ is set to 1 or 0 if it is outside the range [0,1]. 
Step 5: Create a child vector $\mathbf{x}^{\text {new }}$ for each particle by combining a subset of $\mathrm{N}_{\mathrm{var}}-m^{*}$ directly inherited dimensions from $\mathbf{x}_{\mathrm{p}}^{\text {parent }}$ and $\mathrm{m}^{*}$ selected dimensions (via roulette wheel tournament selection) that undergo mutation operation through mapping function based on the means and variances calculated from the particle's solution archive. $m^{*}$ is progressively decreased as follows:

$$
\begin{aligned}
& m^{*}=\operatorname{round}\left(\mathrm{m}_{\text {final }}+\operatorname{rand}\left(m^{\dagger}-\mathrm{m}_{\text {final }}\right)\right) \\
& m^{\dagger}=\operatorname{round}\left(\mathrm{m}_{\text {ini }}-\alpha\left(\mathrm{m}_{\text {ini }}-\mathrm{m}_{\text {final }}\right)\right)
\end{aligned}
$$

-Step 6: The new value of each selected dimension $x_{\mathrm{r}}$ of $\mathbf{x}^{\text {new }}$ is determined by

$$
x_{\mathrm{r}}=h_{\mathrm{x}}+\left(1-h_{1}+h_{0}\right) \cdot x_{\mathrm{r}}^{*}-h_{0}
$$

where $x_{\mathrm{r}}^{*}$ is a randomly generated number with uniform distribution between $[0,1]$, and the term $h$ represents the transformation mapping function defined as follows:

$$
h\left(\bar{x}, s_{1}, s_{2}, x\right)=\bar{x} \cdot\left(1-e^{-x \cdot s_{1}}\right)+(1-\bar{x}) \cdot e^{-(1-x) \cdot s_{2}}
$$

$\mathrm{h}_{\mathrm{x}}, \mathrm{h}_{1}$ and $\mathrm{h}_{0}$ are the outputs of the mapping function calculated for

$$
h_{\mathrm{x}}=h\left(x=x_{\mathrm{r}}^{*}\right), \quad h_{0}=h(x=0), \quad h_{1}=h(x=1)
$$

The shape factors $s_{\mathrm{r} 1}$ and $s_{\mathrm{r} 2}$ of the variable $x_{\mathrm{r}}$ are assigned by using a sequential scheme which accounts for mean and variance of $x_{\mathrm{r}}$, quadratic decrement of $f_{\mathrm{s}}$ from $f_{\mathrm{s} \_ \text {ini }}^{*}$ to $f_{\mathrm{s} \_ \text {final }}^{*}$, and $\Delta d$ in order to exploit the asymmetry of $h$.

Step 7: Stop if the termination criterion is met; else go to Step 2.

The above procedure is illustrated in Figure 1.

\section{Case Study}

Numerical experiments were performed on a computer with an Intel ${ }^{\circledR}$ Core $^{\top \mathrm{TM}} 2$, i7 -3820 central processing unit (CPU), 3.60 GHz processing speed, and 8 GB RAM. The simulation environments MATLAB ${ }^{\circledR}$, MATPOWER (Zimmerman et al, 2011), and DIgSILENT PowerFactory $^{\mathrm{TM}}$ were used to accomplish the implementation aspects and to test the proposed approach. The $Q R$ method is used for full eigenvalue computation. The approach is tested using a slightly modified version of the IEEE New England 39 bus test system (Pai, 2011), which includes two Thyristor-Controlled Series Capacitors (TCSCs), as illustrated in Figure 2. All generators are represented by sub-transient model and equipped with static excitation systems as well as thermal turbine governor systems.

Changes have been made in the system to account for different operating conditions. The approach presented in (Rueda et al, 2011) was used to determine the representative scenarios from probabilistic model based Monte Carlo simulations.

OPCDC is solved by considering the representative scenarios and potential addition of damping controllers at generators $\mathrm{G} 2$ to G10 as well as at both TCSCs. The damping controllers at generators are assumed to have speed as input signals from local generators and are superimposed to the excitation control system, whereas those at TCSCs have line currents as inputs and are superimposed on the device's main control loop, whose output signal is the series compensation susceptance. The parameters of each controller were adjusted considering typical limits, i.e. $K_{S D C} \in[1,100], T_{1}$ and $T_{3} \in[0.2,2], T_{1} / T_{2}$ and $T_{3} / T_{4} \in[1,30]$, whereas the location is decided by using logical variables. Therefore, the search space has 66 dimensions, comprising to 55 continuous variables and 11 two-state discrete variables.

A static penalty scheme is defined for MVMO and the compared algorithms in order to properly consider the fulfillment degree of constraints as well as to ensure fair comparison. The fitness $f^{*}$ is calculated as follows:

$$
f^{*}=f+\sum_{i=1}^{\mathrm{N}_{\mathrm{con}}} \rho_{i} \max \left[0, g_{i}\right]^{2}
$$

where $f$ stands for objective function value, $\mathrm{N}_{\text {con }}$ is the number of constraints, $g_{i}$ denotes the $i$-th constraint, and $\rho$ is the penalty coefficient for each constraint. 

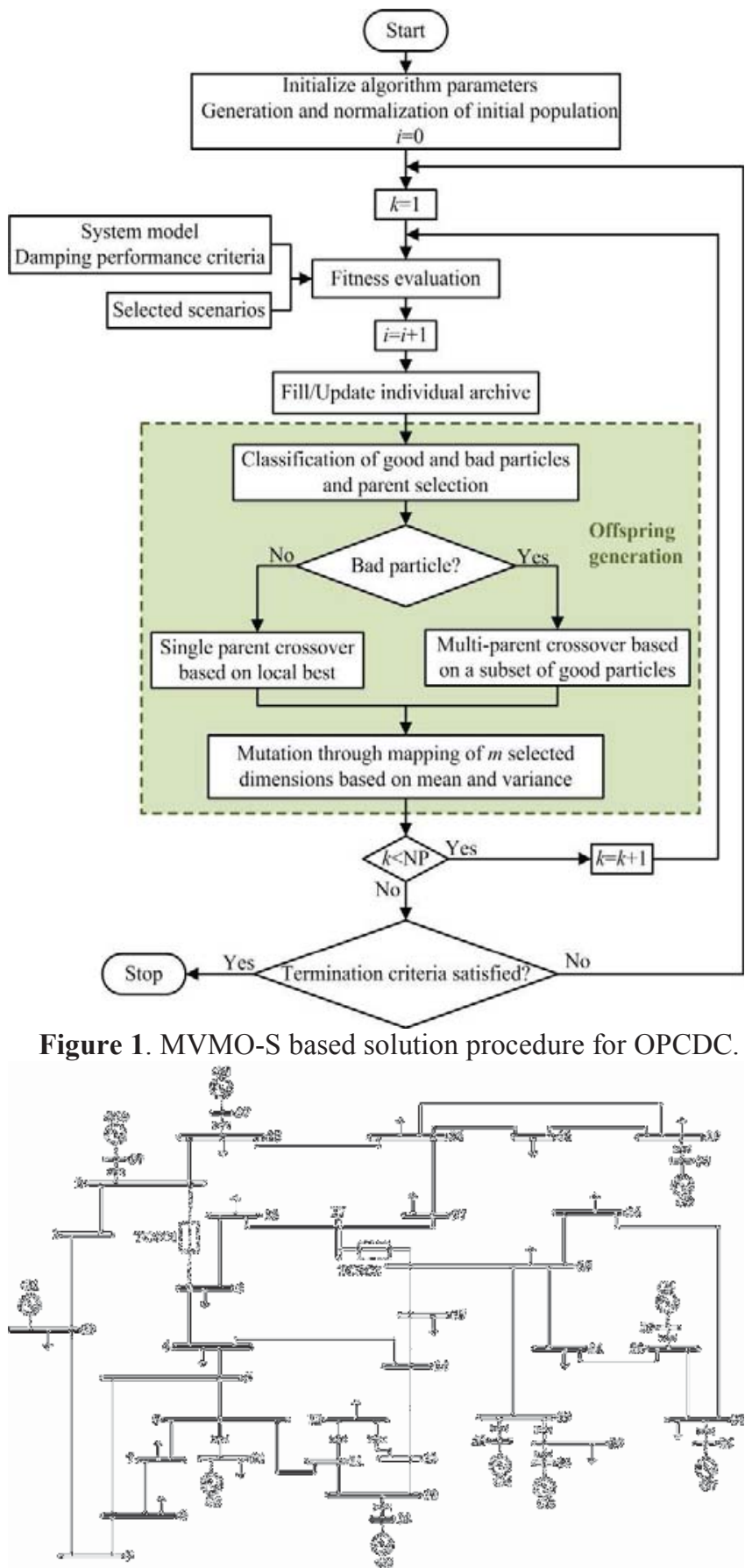

Figure 2. IEEE New England 39 Bus test system.

The average convergence of the fitness, i.e. the OF defined in (1), among 30 independent optimization repetitions is shown in Figure 3, which provides an illustrative comparison between MVMO-S and other emerging metaheuristic optimization algorithms, such as the comprehensive learning particle swarm optimization (CLPSO) (Liang et al, 2011), genetic algorithm with multi-parent crossover (GA-MPC) (Elsayed et al, 2011), differential evolution DE algorithm with adaptive crossover operator (Reynoso-Meza et al, 2011), linearized biogeography-based optimization with re-initialization (LBBO) (Simon et al, 2014), and covariance matrix adaptation evolution strategy (CMA-ES) (Hansen, 2011). By using typical settings provided in the aforesaid references, the goal is to test the suitability of these algorithms as general purpose tools. The stopping criterion was set to 2,000 function evaluations. All algorithms used a population of 40 particles. 


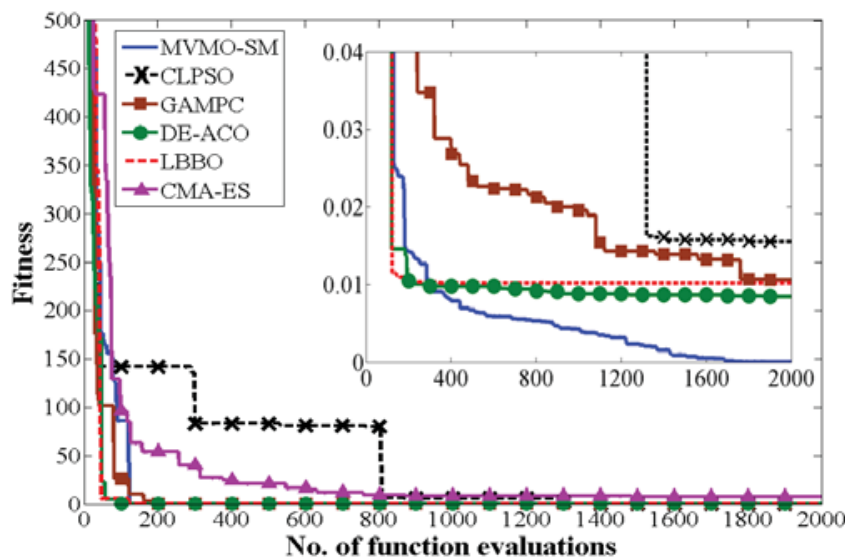

Figure 3. Comparison of average convergence of MVMO-S with other metaheuristic optimization methods.

Table 1. Comparative Performance Statistics

\begin{tabular}{|l|l|l|l|l|l|l|}
\hline \multirow{2}{*}{ Fitness (p.u.) } & \multicolumn{7}{|c|}{ Algorithm } \\
\cline { 2 - 7 } & MVMO-S & CLPSO & GA-MPC & DE-ACO & LBBO & CMA-ES \\
\hline Min. & $1.6691 \times 10^{-7}$ & $8.8302 \times 10^{-3}$ & $5.0714 \times 10^{-3}$ & $4.9339 \times 10^{-3}$ & $4.7855 \times 10^{-3}$ & $8.5323 \times 10^{-5}$ \\
\hline Max. & $1.0659 \times 10^{-4}$ & $4.1739 \times 10^{-2}$ & $1.1517 \times 10^{-2}$ & $9.8407 \times 10^{-3}$ & $1.1517 \times 10^{-2}$ & $6.9723 \times 10^{+1}$ \\
\hline Mean & $1.5199 \times 10^{-5}$ & $1.5509 \times 10^{-2}$ & $1.0590 \times 10^{-2}$ & $8.4228 \times 10^{-3}$ & $1.0178 \times 10^{-2}$ & $0.7809 \times 10^{+1}$ \\
\hline Std. & $2.9867 \times 10^{-5}$ & $1.1341 \times 10^{-2}$ & $2.1137 \times 10^{-3}$ & $1.3087 \times 10^{-3}$ & $2.2180 \times 10^{-3}$ & $2.1906 \times 10^{+1}$ \\
\hline $\begin{array}{l}\text { Average } \\
\text { execution } \\
\text { time (min) }\end{array}$ & 26.2537 & 26.6231 & 29.7757 & 26.6538 & 25.9259 & 26.5142 \\
\hline
\end{tabular}

From Figure 3, note the excellent performance of MVMO-SM in terms of both convergence speed and the minimum reached, since after the first 1,600 function evaluations, it is able to locate the global optimal solution in the search space (when the thresholds for damping performance are reached, i.e. $\mathrm{OF}=0$ ) without being trapped in a local optimum.

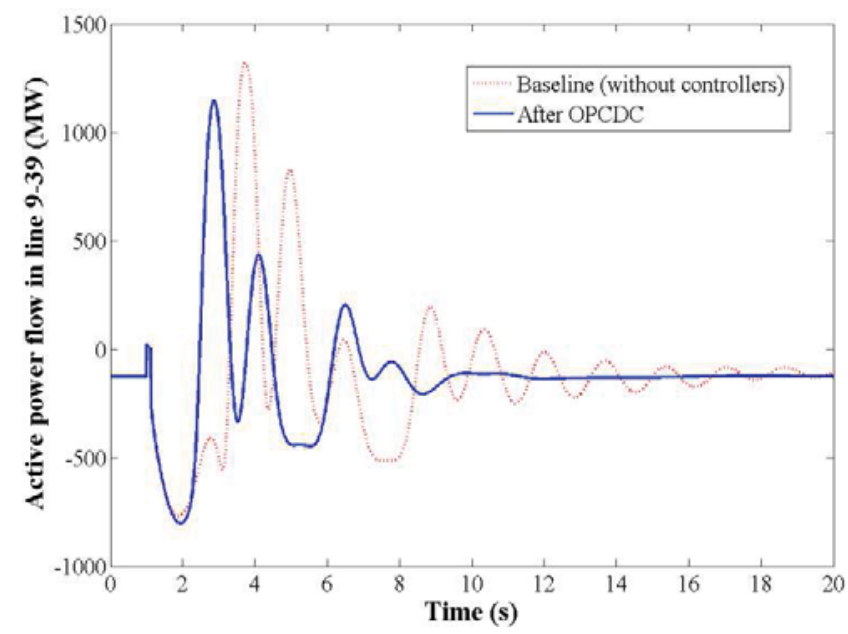

Figure 4. Active power flow in line 9-39.

In the figure, the high nonzero values of fitness observed at the beginning of the convergence progress are due to fact that the global damping factor measure is lower that the threshold, cf. (1). It is also noticed in the figure that all algorithms are capable of finding solutions that entail satisfying the damping factor threshold (i.e. obtaining fitness values that are considerably smaller than those obtained at the beginning of the search process), but finding solutions that simultaneously allow satisfying both damping factor and damping ratio thresholds (i.e. $\mathrm{OF}=0$ ) is what make the difference in the performance of the algorithms. A statistical survey of the achieved fitness values for all optimization repetitions is given in Table I, where the outstanding performance of MVMO-S can be more clearly appreciated by comparing different statistical attributes. The table also summarizes the average 
execution time of the optimization task for all algorithms. It can be seen that there are some slight differences, which are due to inherent algorithmic characteristics of each method.

Time domain simulation was also performed to confirm the effectiveness of the obtained results. For this purpose, the simulation was conducted for one of the scenarios considered in the OPCDC by applying a three-phase fault at bus 15 at $t=1 \mathrm{~s}$, with a duration of $100 \mathrm{~ms}$. Figure 4 presents the oscillograms corresponding to the active power flow of transmission line $9-39$, showing the enhancement of damping performance.

\section{Conclusions}

This paper presents a metaheuristic based approach to tackle the problem of optimal placement and coordinated tuning of power system supplementary damping controllers. The optimization task is solved via MVMO-S. Numerical results attest the outstanding performance of the proposed MVMO-SM in terms of convergence behaviour and lowest statistical attributes associated to optimization repetition. The application of the approach to a real large-size power system is currently being carried out in order to further ascertain its effectiveness. Moreover, the proposed approach can be extended to include other devices in which a damping controller can be added. Ongoing research is also being conducted to evaluate the performance of MVMO-S when solving other power system optimization problems, like the optimal active-reactive power dispatch problem in wind-hydro-thermal systems considering uncertainties and security constraints and the optimal dynamic transmission expansion planning.

\section{References}

A. Karimpour, R. Asgharian, and O. P. Malik. 2005. Determination of PSS location based on singular value decomposition. International Journal of Electrical Power \& Energy Systems, vol. 27, no 8, pp. 535-541.

D. Simon, M.G.H. Omran, M. Clerc. 2014. Linearized Biogeography-Based Optimization with Reinitialization and Local Search. Information Sciences, vol. 267, no. 20, pp. 140-157.

F.L. Pagola, I.J. Perez-Arriaga, and G.C. Verghese. 1989. n sensitivities, residues and participations: applications to oscillatory stability analysis and control. IEEE Transactions on Power Systems, vol. 4, no. 1, pp. 278 - 285.

G. Reynoso-Meza, J. Sanchis, X. Blasco, and J.M. Herrero. 2011. Hybrid DE algorithm with adaptive crossover operator for solving real-world numerical optimization problems. Proc. IEEE Congress on Evolutionary Computation, New Orleans, USA, pp. $1551-1556$

H.F. Wang, F.J. Swift, and M. Li. 1997. Indices for selecting the best location of PSSs or FACTS-based stabilizers in multimachine power systems: a comparative study. IEE Proceedings Generation, Transmission and Distribution, vol. 4, no. 2, pp. 155 159.

J.J. Liang, A.K. Qin, P.N. Suganthan, S. Baskar. 2006. Comprehensive learning particle swarm optimizer for global optimization of multimodal functions. IEEE Transactions on Evolutionary Computation, vol. 10, no. 3, pp. 281- 295.

J.L. Rueda, J.C. Cepeda, and I. Erlich. 2014. Probabilistic approach for optimal placement and tuning of power system supplementary damping controllers. IET Generation, Transmission \& Distribution, vol. 8, no.11, pp.1831-1842.

J.L. Rueda, and I. Erlich. 2013. Mean-Variance Mapping Optimization for the Solution of Power System Problems. Proceedings of the 17th International Conference on Intelligent System Applications to Power Systems, pp. 1-8. Tokyo, Japan.

K. Sebaa, H. Gueguen, and M. Boudour. 2010. Mixed integer non-linear programming via the cross-entropy approach for power system stabilisers location and tuning. IET Generation, Transmission \& Distribution, vol. 4, no. 8, pp. 928-939.

K. Sebaa, and M. Boudour. 2009. Optimal locations and tuning of robust power system stabilizer using genetic algorithms. Electric Power Systems Research, vol. 79, no. 2, pp. 406-416.

M. Eslami, H. Shareef, A. Mohamed, and M. Khajehzadeh. 2012. An efficient particle swarm optimization technique with chaotic sequence for optimal tuning and placement of PSS in power systems. International Journal of Electrical Power and Energy Systems, vol. 43, no. 1, pp. 1467-1478.

M. A. Pai, 1989. Energy Function Analysis for Power System Stability. Kluwer Academic Publishers.

N. Hansen. The CMA evolution strategy: A tutorial. [Online]: http://www.lri.fr/hansen/cmatutorial.pdf, accessed April 2014.

R. D. Zimmerman, C. E. Murillo-Sánchez, and R. J. Thomas. 2011. MATPOWER: Steady-State Operations, Planning and Analysis Tools for Power Systems Research and Education. IEEE Transactions on Power Systems, vol. 26, no. 1, pp. 12-19.

S.M. Elsayed, R.A. Sarker, D.L. Essam. 2011. GA with a new multi-parent crossover for solving IEEE-CEC2011 competition problems. Proc. IEEE Congress on Evolutionary Computation, New Orleans, USA, , pp. 1034-1040.

Z. Wang, C.Y. Chung, K.P. Wong, and C. T. Tse. 2008. Robust power system stabilizer design under multi-operating conditions using differential evolution. IET Generation, Transmission \& Distribution, vol. 2, no. 5, pp. 690-700.

\section{Biographical notes}

J. L. Rueda Torres received the Electrical Engineer diploma from the Escuela Politécnica Nacional, Quito, Ecuador, in 2004, and the Ph.D. degree in electrical engineering from the Universidad Nacional de San Juan, San Juan, Argentina, in 2009. From September 2003 till February 2005 , he worked in Ecuador, in the fields of industrial control systems and electrical distribution networks operation and planning. Between 2010 and 2014 he worked as a postdoctoral research associate at the Institute of Electrical Power Systems, University of Duisburg-Essen, Germany, where he is currently pursuing the pursuing the 'Habilitation' 
(qualification) postdoctoral degree. Currently, he is working as an assistant professor for Intelligent Electrical Power Grids at the Department of Electrical Sustainable Energy, Technical University Delft, Netherlands. Dr. Rueda is vice-chair of the Working Group on Modern Heuristic Optimization (WGMHO) under the IEEE PES Power System Analysis, Computing, and Economics Committee. His research interests include power system stability and control, system identification, power system planning, probabilistic and artificial intelligence methods.

Francisco M. Gonzalez-Longatt is currently a Lecturer in Electrical Power System at Electrical Power System in School of Electronic, Electrical and Systems Engineering en Loughborough University. His academic qualifications include first Class Electrical Engineering of Instituto Universitario Politécnico de la Fuerza Armada Nacional, Venezuela (1994), Master of Business Administration (Honors) of Universidad Bicentenaria de Aragua, Venezuela (1999) and PhD in Electrical Power Engineering from the Universidad Central de Venezuela (2008). He is former associate professor (1995-2009) and Chair (1999-2001) of the Department of Electrical Engineering of Universidad Nacional Politécnico de la Fuerza Armada Nacional, Venezuela (1995-2009). He is a former academic staff of Department of Aerospace, Electrical and Electronic Engineering at University of Coventry where he started as Lecturer in Electrical Engineering in 2012 and promoted to Senior Lecturer in Electrical Engineering in 2013. He was formerly with the School of Electrical and Electronic Engineering, The University of Manchester as Postdoctoral Research Associate (2009-2011). He is Vice-President of Venezuelan Wind Energy Association, Senior Member of the IEEE, member of The Institution of Engineering and Technology - The IET (UK) and member of International Council on Large Electric Systems -CIGRE. His research interest includes innovative (operation/control) schemes to optimize the performance of future energy systems.

Received March 2015

Accepted July 2015

Final acceptance in revised form July 2015 